\title{
Epigenetic inactivation of SFRP genes allows constitutive WNT signaling in colorectal cancer
}

\author{
Hiromu Suzuki ${ }^{1,2}$, D Neil Watkins ${ }^{1}$, Kam-Wing Jair ${ }^{1}$, Kornel E Schuebel ${ }^{1}$, Sanford D Markowitz ${ }^{3,4}$, Wei Dong Chen ${ }^{4}$, \\ Theresa P Pretlow ${ }^{5}$, Bin Yang ${ }^{1}$, Yoshimitsu Akiyama ${ }^{1}$, Manon van Engeland ${ }^{6}$, Minoru Toyota ${ }^{2,7,8}$, Takashi Tokino ${ }^{7}$, \\ Yuji Hinoda ${ }^{9}$, Kohzoh Imai ${ }^{2}$, James G Herman ${ }^{1} \&$ Stephen B Baylin $^{1}$
}

\begin{abstract}
Aberrant WNT pathway signaling is an early progression event in $90 \%$ of colorectal cancers ${ }^{1}$. It occurs through mutations mainly of $A P C$ and less often of CTNNB1 (encoding $\beta$-catenin) ${ }^{1-3}$ or AXIN2 (encoding axin-2, also known as conductin) ${ }^{4}$. These mutations allow ligandindependent WNT signaling that culminates in abnormal accumulation of free $\beta$-catenin in the nucleus ${ }^{1-3}$. We previously identified frequent promoter hypermethylation and gene silencing of the genes encoding secreted frizzledrelated proteins (SFRPs) in colorectal cancer ${ }^{5}$. SFRPs possess a domain similar to one in the WNT-receptor frizzled proteins and can inhibit WNT receptor binding to downregulate pathway signaling during development ${ }^{6-10}$. Here we show that restoration of SFRP function in colorectal cancer cells attenuates WNT signaling even in the presence of downstream mutations. We also show that the epigenetic loss of SFRP function occurs early in colorectal cancer progression and may thus provide constitutive WNT signaling that is required to complement downstream mutations in the evolution of colorectal cancer.
\end{abstract}

Figure 1 Colorectal cancer cells express various WNTs and the WNT signaling pathway can be suppressed by SFRPs. (a) RT-PCR analysis of WNT genes in colorectal cancer cell lines. Gene symbols are indicated on the left. PCR was done by using cDNA prepared with (+) or without $(-)$ reverse transcriptase (RT). GAPD is also shown as a control.

(b) Reporter gene assays by using a TCF-LEF-responsive reporter (pGL3OT) and a negative control with a mutated TCF-LEF binding site (pGL3OF) to analyze CRT. Results, expressed relative to a value of 1.0 for cells transfected with empty vector, are the means of three replications, and error bars represent standard deviations. (c) Schematic representation of SFRP2 deletion mutants. (d,e) Effects of SFRP2 mutants on CRT in HCT116 and SW480 cells. a

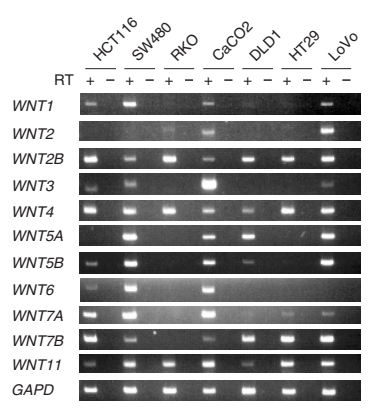

b
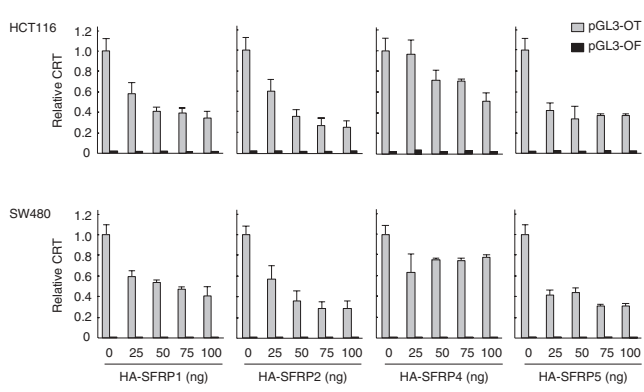

C

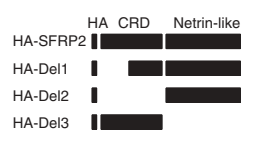

d

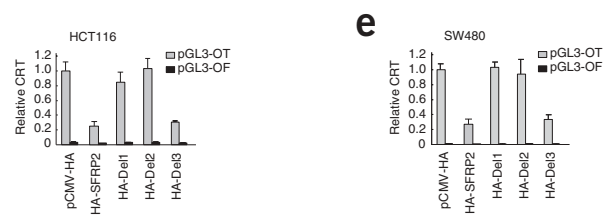

${ }^{1}$ Division of Tumor Biology, The Sidney Kimmel Comprehensive Cancer Center, Johns Hopkins Medical Institutions, 1650 Orleans Street, Baltimore, Maryland 21231, USA. ${ }^{2}$ First Department of Internal Medicine, Sapporo Medical University, Sapporo, Japan. ${ }^{3}$ Howard Hughes Medical Institute, Cleveland, Ohio 44106, USA. ${ }^{4}$ Department of Medicine and Ireland Cancer Center, Case Western Reserve University and University Hospitals of Cleveland, Cleveland, Ohio 44106, USA. ${ }^{5}$ Department of Pathology, Case Western Reserve University, Cleveland, Ohio 44106, USA. ${ }^{6}$ Department of Pathology, University of Maastricht, 6200 MD Maastricht, the Netherlands. ${ }^{7}$ Department of Molecular Biology, Cancer Research Institute, Sapporo Medical University, Sapporo, Japan. ${ }^{8}$ PRESTO, JST, Kawaguchi, Japan. ${ }^{9}$ Department of Clinical Laboratory Science, Yamaguchi University School of Medicine, Yamaguchi, Japan. Correspondence should be addressed to S.B.B. (sbaylin@jhmi.edu). 
To begin addressing the role of epigenetic silencing of SFRP genes, we first looked for expression of WNT proteins in colorectal cancer. In all seven colorectal cancer cell lines examined, we found expression of 3 or more of 11 members of the WNT family (Fig. 1a), a result consistent with previous studies in primary and cultured colorectal cancers $^{11-13}$. Next, we asked whether SFRPs could downregulate WNT signaling in the colorectal cancer cell lines HCT116 and SW480, which harbor mutations in CTNNB1 and $A P C$, respectively ${ }^{2,3}$. Indeed, equal
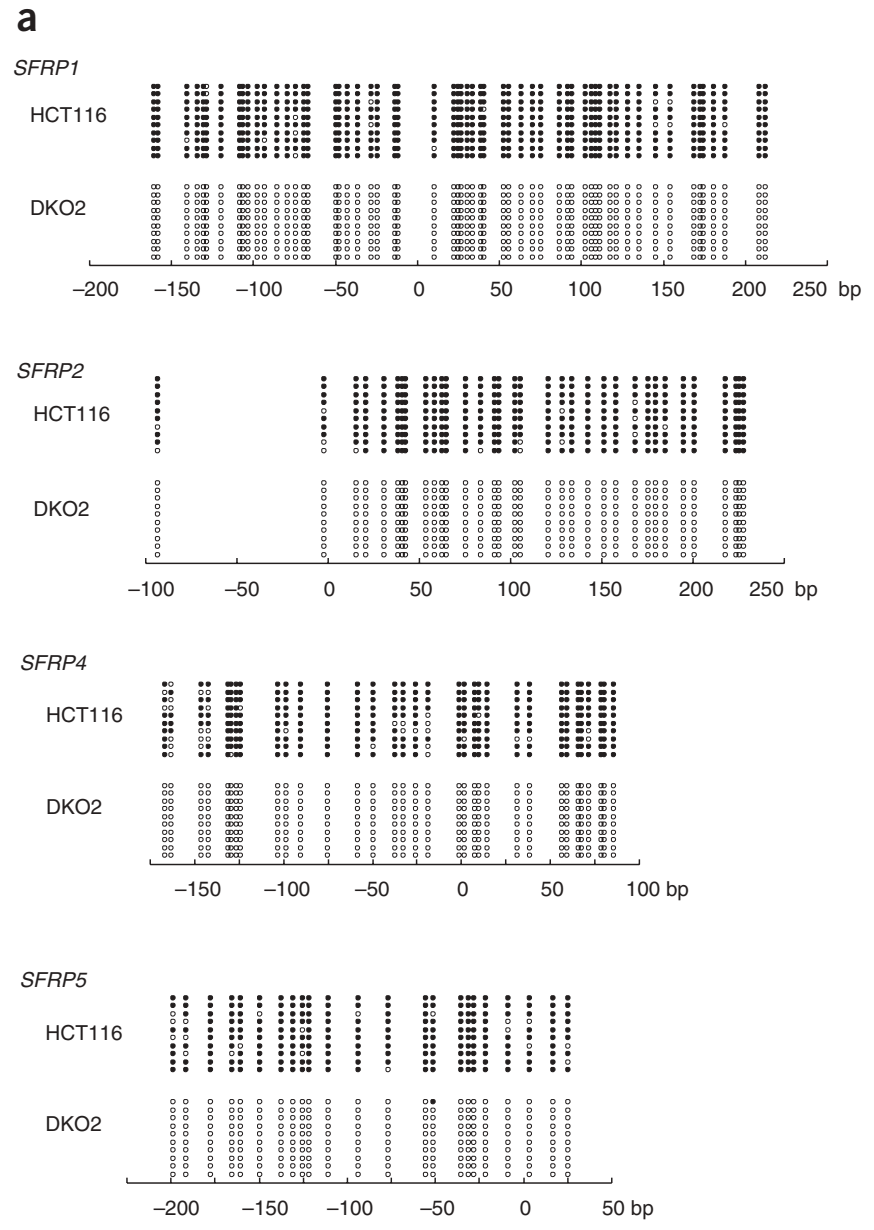

b

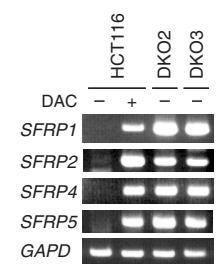

C

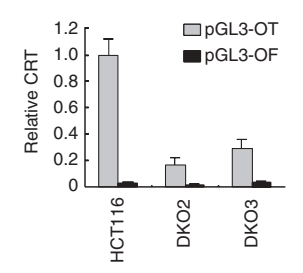

Figure 2 Demethylation and re-expression of SFRP genes and suppression of the WNT pathway in DKO cells. (a) Bisulfite sequencing analysis of SFRP genes in wild-type HCT116 and DKO cells. Open and filled circles represent unmethylated and methylated $\mathrm{CpG}$ sites, respectively, and each row represents a single clone. Locations of $\mathrm{CpG}$ sites relative to transcription start sites are shown below. (b) RT-PCR analysis of SFRP genes in HCT116 and two different clones of the DKO cells. HCT116 cells treated with 5-aza$2^{\prime}$-deoxycytidine (DAC) for $72 \mathrm{~h}$ were used as a control for the expression of the SFRP genes. (c) CRT analysis in wild-type HCT116 and DKO cells. Bars and conditions are the same as in Figure 1. expression of hemagglutinin (HA)-tagged SFRP1, SFRP2 and SFRP5, and, to a lesser extent, SFRP4, suppressed $\beta$-catenin-T-cell factor (TCF)-regulated transcription (CRT) activity from a reporter responsive to TCF and lymphoid enhancer-binding factor (LEF) in a dosedependent manner (Fig. $\mathbf{1 b}$ and Supplementary Fig. 1 online). Notably, SFRP4 is less frequently hypermethylated in colorectal can$\operatorname{cer}^{5}$ and has the least homology with other family members ${ }^{14}$. SFRP1 is thought to silence ligand-dependent WNT signaling by binding of the cysteine rich-domain (CRD) to WNT proteins, thus preventing interaction with FRZ receptors ${ }^{15-18}$. When we transfected SFRP2 deletion mutants lacking either the cysteine-rich domain (CRD) or the netrin-like domain (Fig. 1c and Supplementary Fig. 1 online), the CRD was necessary and sufficient to repress WNT signaling in both HCT116 and SW480 cells (Fig. 1d,e).

To examine the possibility that re-expression of epigenetically silenced endogenous SFRP genes might have an effect similar to that of their exogenous overexpression, we used HCT116 cells in which the DNA methyltransferase genes DNMT1 and DNMT3B are genetically disrupted (DKO cells) and gene silencing is abrogated ${ }^{19-21}$. SFRP1, SFRP2, SFRP4 and SFRP5 are hypermethylated and silenced in wildtype HCT116 cells, whereas DKO cells had virtually complete demethylation, re-expression of each SFRP gene (Fig. 2a,b) and marked reductions in canonical WNT signaling relative to wild-type HCT116 cells (Fig. 2c). a

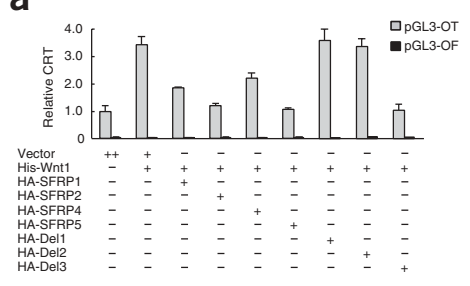

b

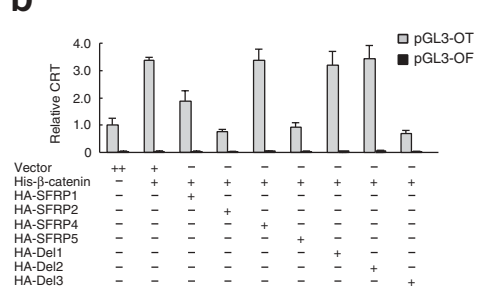

e
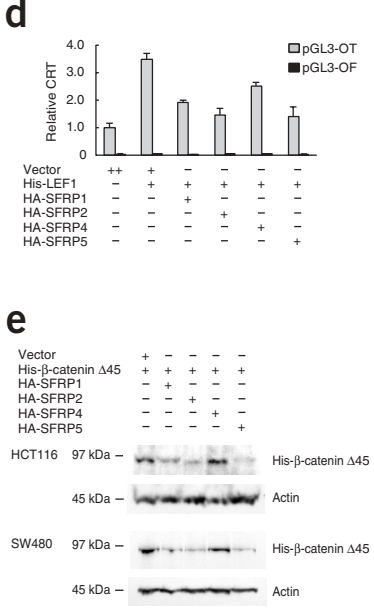

C

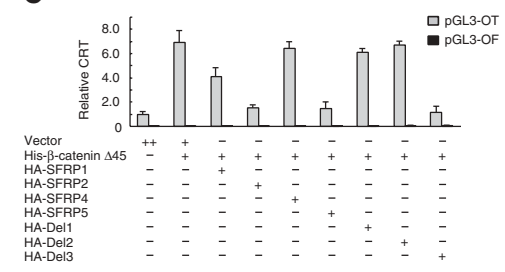

Figure 3 Upregulation of the canonical WNT pathway by WNT1, $\beta$-catenin and LEF1, which can be suppressed by SFRPs in colon cancer cells. (a) CRT analysis in HCT116 cells cotransfected with His-WNT1 and each HA-SFRP construct, plus reporter assay genes. (b) CRT analysis in HCT116 cells cotransfected with His-tagged wild-type $\beta$-catenin and each HA-SFRP construct. (c) CRT analysis in HCT116 cells cotransfected with His-tagged mutant $\beta$-catenin ( $\beta$-catenin $\triangle 45$ ) and each HA-SFRP construct. (d) CRT analysis in HCT116 cells cotransfected with His-tagged full-length LEF1 and each HA-SFRP construct. (e) Western-blot analysis of exogenous His- $\beta$ catenin $\triangle 45$ in HCT116 and SW480 cells cotransfected with HA-SFRPs or an empty vector. Actin is also shown as a control for protein loading. 
These results raise the possibility that, even in the presence of activating mutations downstream of the FRZ receptor, the WNT pathway can be further constitutively activated at the ligand level. Indeed, we find that transfection of WNT1 into HCT116 cells increased reporter activation threefold, suggesting that these cells with mutant CTNNB1 retained sensitivity to ligand-driven signaling (Fig. 3a). Transfection of SFRP1, SFRP2, SFRP5 or, to a lesser extent, SFRP4, suppressed this signaling to near-basal levels, and only SFRP2 deletion mutants retaining the CRD showed suppression activity (Fig. 3a). Thus, HCT116 cells retained the ability to respond to exogenous WNT1 ligand, and this effect could be inhibited by SFRPs.

We next investigated whether increasing levels of cellular $\beta$-catenin could further increase its nuclear activity in colorectal cancer cells. In transfections of wild-type or mutant CTNNB1 ( $\beta$-catenin $\Delta 45)$, with or without SFRP genes, wild-type CTNNB1 induced CRT by a factor of $\sim 3.5$ (Fig. 3b) and, notably, the mutant induced CRT by a factor of 7 (Fig. 3c), and SFRP1, SFRP2 and SFRP5 inhibited both wild-type and mutant $\beta$-catenin (Fig. 3b,c). $\beta$-catenin $\Delta 45$ is encoded by a CTNNB1 allele with a deletion of $3 \mathrm{bp}$, resulting in the absence of residue 45 of $\beta$-catenin; this protein is thought to be resistant to phosphorylation by the APC, GSK3 $\beta$ and axin-conductin complex, rendering it constitutively active ${ }^{3}$. We obtained similar results in SW480 cells (data not shown). Lymphoid enhancer-binding factor-1 (LEF1), a reported target of CRT, forms a complex with $\beta$-catenin in the nucleus to activate transcription of target genes. A full-length isoform is selectively expressed in colon cancers $^{22}$. We obtained results similar to those described above in HCT116 cells cotransfected with a full-length LEF1 vector and SFRP genes (Fig. 3d). Thus, SFRP can potentially inhibit the entire canonical WNT pathway in colon cancer cells.

Phosphorylation of free $\beta$-catenin by the APC-axin-GSK3 $\beta$ complex silences canonical WNT signaling by stimulating ubiquitination of the protein, thus decreasing its total cellular levels and nuclear localization ${ }^{1,23}$. Nuclear presence of $\beta$-catenin, especially, is a hallmark of active WNT signaling ${ }^{1,24}$. In this regard, we find that overexpressing SFRP1, SFRP2 and SFRP5 decreased levels of an overexpressed tagged wild-type or mutant $\beta$-catenin protein in HCT-116 and SW480 cells (Fig. 3e). This finding may be especially surprising for the $\beta$-catenin $\Delta 45$ form, as it is thought to be constitutively stabilized through mutation of a serine site that must be

Figure 4 SFRPs suppress $\beta$-catenin and CRT target gene expression and induce apoptosis. (a) Staining of endogenous $\beta$-catenin (green) and exogenous HA-SFRPs (red) in SW480 cells transiently transfected with HASFRPs. White arrows indicate transfected cells. Cells not expressing transfected SFRP1, SFRP2 and SFRP 5 have abundant staining of $\beta$-catenin (green) in the cytoplasm, including in the nucleus. Cells positive for expression of transfected SFRP1, SFRP2 and SFRP5 (red) have very little or no staining for $\beta$-catenin, but the protein is retained in cells expressing exogenous SFRP4. (b) Staining of endogenous $\beta$-catenin in HCT116 cells. (c) Staining of $\beta$-catenin in DKO cells. Wild-type cells show prominent $\beta$ catenin staining (green) in cytoplasm, at discrete cell membrane sites for adjacent cells representing adherens junctions and in the nuclei (white arrows). In DKO cells, staining is retained in the cytoplasm and at the membrane junctions but is essentially absent from the nuclei. (d) Northernblot analysis of MYC in SW480 cells transfected with pcDNA3.1/HisASFRPs or an empty vector and selected for $14 \mathrm{~d}$ with G418. GAPD is shown as a control. (e) Northern-blot analysis of MYC in HCT116 and DKO cells. $G A P D$ is shown as a control. EtBr, ethidium bromide stain, is shown as a loading amount control. (f) Representative results of flow cytometry assay in HCT 116 cells. Cells at $72 \mathrm{~h}$ after transfection are shown. Apoptotic cells are indicated as the sub-G1 fraction. (g) Summarized quantitative results of the apoptosis assay in HCT116 cells. Results are the means of three replications, and error bars represent standard deviations. phosphorylated to signal ubiquitination ${ }^{1,3}$. But WNT signaling controls phosphorylation status through several different crucial serine and threonine sites, any of which, when mutated, contributes to increased WNT effects ${ }^{25}$, and individual colorectal cancers with CTNNB1 mutations contain only one mutant site at a time. Thus, in the setting of such individual mutations, there may still be selective tumorigenic advantage for increased WNT signaling.

We next examined endogenous $\beta$-catenin levels in HCT116 and SW480 cells transfected with SFRP genes. Immunofluorescence analysis showed decreased levels of overall cytoplasmic and nuclear $\beta$-catenin in SW480 cells, which contain an APC mutation, with overexpression of SFRP1, SFRP2 and SFRP5, but not of SFRP4 (Fig. 4a); we observed similar results in HCT116 (data not shown). Notably, we observed a specific loss of nuclear $\beta$-catenin, with retention of cytoplasmic and membrane-localized content, in DKO cells that reexpressed SFRP genes and contained an endogenously mutated CTNNB1 gene (Fig. $\mathbf{4 b}, \mathbf{c}$ ).

What might be the functional consequences for colorectal cancer cells of WNT-pathway suppression by SFRPs? The oncogene MYC is a well known target gene of canonical WNT signaling ${ }^{26}$. We found that stable overexpression of SFRP1, SFRP2 and SFRP5 in SW480 cells resulted in substantial downregulation of MYC mRNA after 2 weeks of selection (Fig. 4d). DKO cells also showed substantial downregulation of MYC, which is concordant with the results of our reporter assay and $\beta$-catenin staining experiment (Fig. 4e).

We next looked at the effects of SFRPs on the growth and apoptosis of colorectal cancer cells. Decreased colony formation occurred in cells stably overexpressing each of the SFRPs (Supplementary Fig. 2 online), including, unexpectedly, SFRP4, which has the weakest WNT signaling inhibition when overexpressed. Notably, however, HCT116 and SW480 cells showed an induction of apoptosis that correlated a

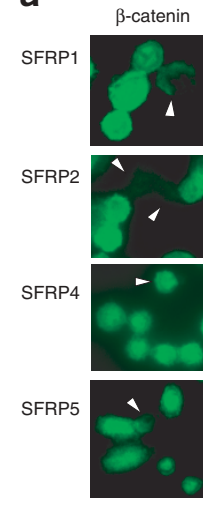

d

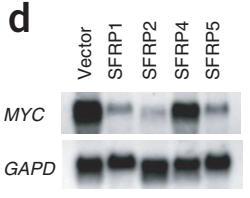

e

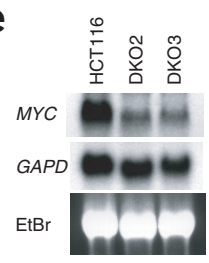

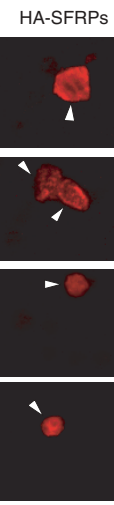

$f$
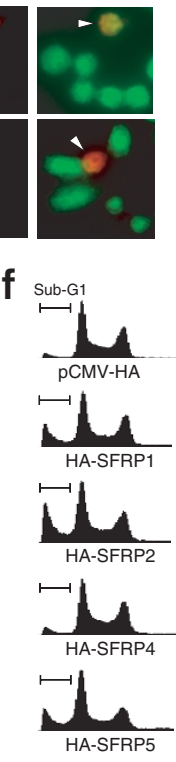

b

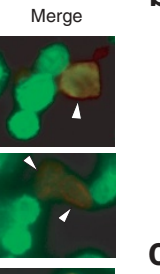

C

g

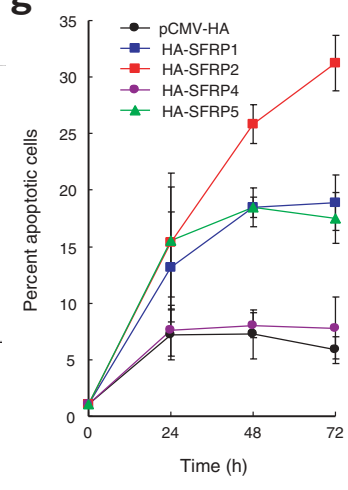

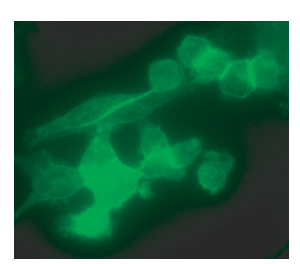

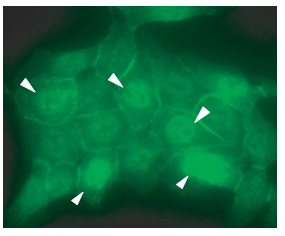


perfectly with the differential effects of the SFRPs on WNT activity. HCT116 cells transiently transfected with SFRP1, SFRP2 and SFRP5, but not with SFRP4, had elevated apoptosis responses (Fig. $\mathbf{4 f , g}$ ), and SW480 cells showed similar effects (data not shown).

We next tried to place the in vitro data in the context of the progression of primary colorectal cancer. We first analyzed APC mutations and SFRP methylation in 91 primary colorectal cancer tumors. Sixtythree tumors $(69.2 \%)$ showed mutations in $A P C$ and 81 tumors (89\%) showed methylation of both SFRP1 and SFRP2. Mutations of $A P C$ in primary colorectal cancer tumors frequently then coincided a

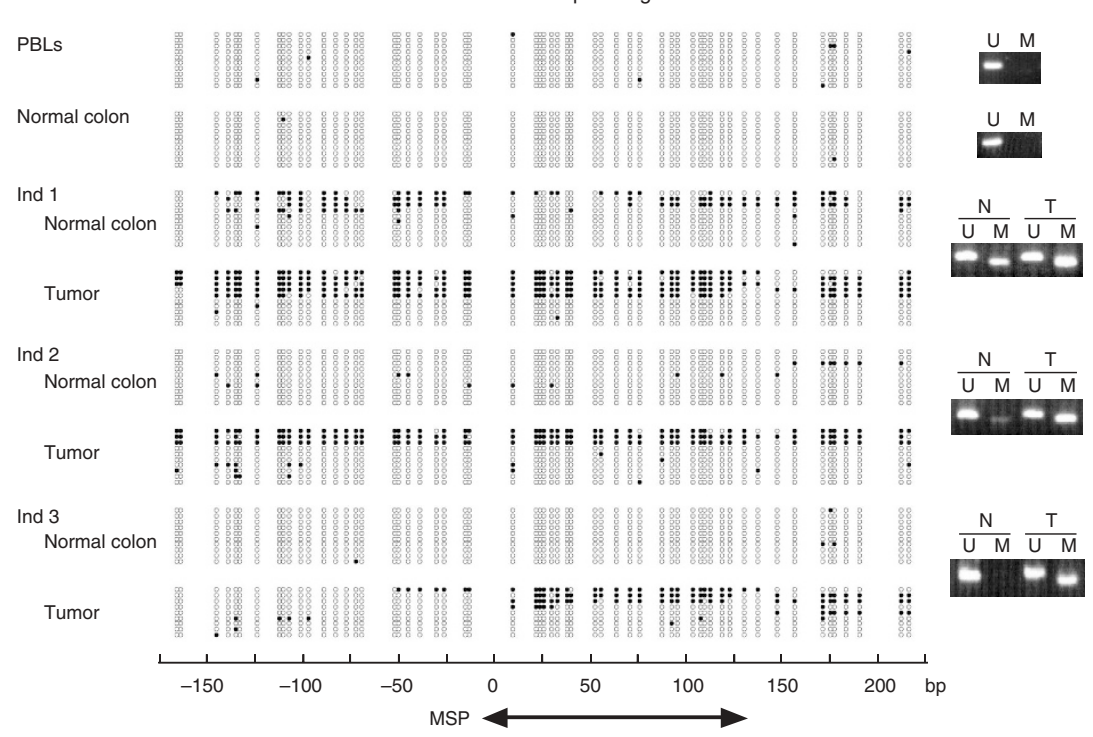

b

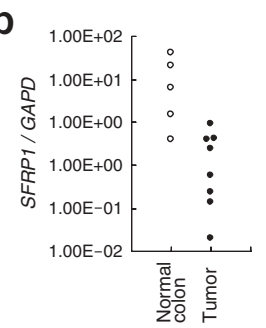

e

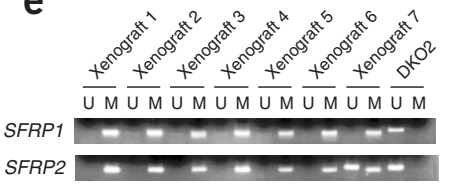

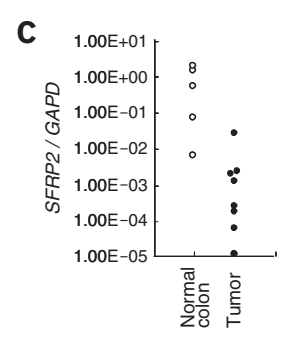

d
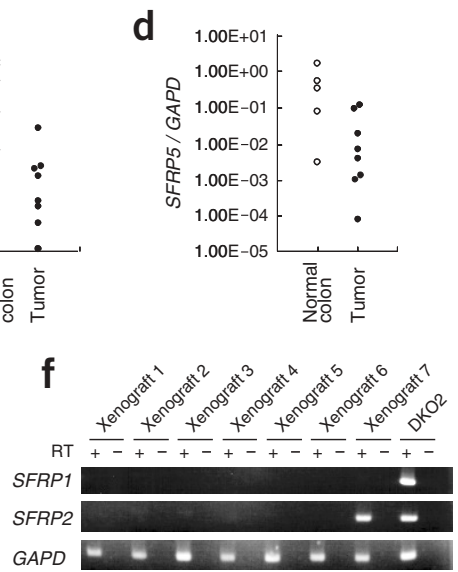

Figure 5 Methylation and expression analysis of SFRP genes in primary colorectal cancers (a) Bisulfite sequencing analysis of SFRP1 in peripheral blood lymphocytes (PBLS), normal colon tissue, and paired normal counterparts $(\mathrm{N})$ and tumor tissues $(\mathrm{T})$ from three individuals with colorectal cancer. Open and filled circles represent unmethylated (U) and methylated (M) CpG sites, respectively, and each row represents a single clone. The location of each $\mathrm{CpG}$ site relative to the transcription start site and a region analyzed by the MSP analysis are shown below. MSP results from the same samples are shown on the right. (b-d) Summarized results of real-time RT-PCR of SFRP1, SFRP2 and SFRP5 in a series of normal colon mucosa from individuals with colorectal cancer or colorectal adenoma $(n=5)$ and primary colorectal cancers that showed SFRP methylation $(n=8)$. Results are normalized for internal GAPD expression. (e) MSP analysis of SFRP1 and SFRP2 in xenograft samples from colorectal cancer. DKO2 cells served as unmethylated controls. (f) RT-PCR analysis of SFRP1 and SFRP2 in colorectal cancer xenografts. DKO2 cells served as positive controls for the expression. GAPD is also shown as a control. individuals with colorectal cancer, although MSP detected partial methylation of SFRP1 in both the normal counterpart and the tumor tissue, bisulfite sequencing showed that almost the entire promoter region was densely hypermethylated in half the clones analyzed from the tumor. The unmethylated clones probably came from contaminating normal stromal cells. In contrast, the normal counterpart showed heterogeneous patterns; in particular, $\mathrm{CpG}$ sites around the transcription start site did not show dense hypermethylation. Normal tissue samples from individuals with colorectal cancer that showed weak or no methylation signals by MSP had methylation of only a few CpG sites and lacked dense methylation near the transcription start site (Fig. 5a and Supplementary Fig. 3 online).

We correlated the hypermethylation and expression of SFRP genes in normal colon mucosa from individuals with colorectal cancer or colorectal adenoma $(n=5)$ and primary tumor tissues harboring hypermethylated SFRP genes $(n=8)$ and found, by real-time PCR, substantial overall decreases in the expression levels of SFRP1, SFRP2 and SFRP5 (Fig. 5b-d). The presence of normal cells in these tumors could contribute to an underestimation of both the promoter hypermethylation and expression of these genes in these studies. Indeed, xenografts into scid mice of fresh specimens from seven individuals with colorectal cancer, which contained no normal human cells, had complete methylation of SFRP1 and lacked expression by RT-PCR (Fig. 5e). We observed similar results for SFRP2 in six of the seven individuals, and only one tumor with partial methylation expressed the gene (Fig. 5f).

Mutational inactivation of the APC tumor suppressor occurs early, in premalignant lesions, in colorectal cancer progression ${ }^{1}$. We now find (Fig. 6a,c), in 15 samples from 11 individuals, frequent methylation of SFRP1 and SFRP2 (14 of 15 and 13 of 15, respectively) in one of the earliest lesions, monoclonal aberrant crypt foci (ACF) ${ }^{27}$ that usually lack APC mutations ${ }^{28}$. As is true in colorectal cancers ${ }^{5}$, SFRP4 and SFRP5 were methylated in fewer of these samples (6 of 15 
and 8 of 15, respectively). We also observed similar results in 30 samples of a later benign progression stage of colorectal cancer, colorectal adenomas, with higher methylation frequencies for $S F R P 1, S F R P 2$ and SFRP5 and a frequency of only $17 \%$ for SFRP4 (Fig. $\mathbf{6 b}, \mathbf{d}$ ).

We propose, from our findings, that epigenetic downregulation of SFRP genes may be instrumental, through its contribution to previously unrecognized constitutive WNT ligand signaling and cell resistance to apoptosis, in the appearance of early premalignant changes that predispose to colorectal cancer. Although these changes alone may not be sufficient to induce colorectal cancer, the cells involved would then be particularly sensitive to malignant changes that might be initiated by mutations in APC or CTNNB1. The complementary effects of these epigenetic and genetic changes for WNT pathway activation then persist throughout colorectal cancer progression. In addition to these important biologic possibilities, hypermethylation patterns of SFRP genes may provide a potentially useful marker system for predicting the risk of colonic neoplasia. Finally, modulation of WNT protein-driven cell growth, through strategies such as monoclonal antibody blockade ${ }^{29}$ or reversal of SFRP silencing, may represent potential targets for colorectal cancer prevention and treatment.

\section{METHODS}

Cell culture, drug treatment and tissue samples. We cultured the human colorectal cancer lines HCT116 and SW480 in McCoy5A medium (HyClone) supplemented with $10 \%$ bovine calf serum (HyClone). We cultured HCT116 cells with genetic disruption of DNMT1 and DNMT3B as described ${ }^{19}$. We treated HCT116 cells with $2 \mu \mathrm{M} 5$-aza-2'-deoxycidine for $72 \mathrm{~h}$ as described ${ }^{5}$. We obtained primary colorectal cancer samples and colorectal adenomas from surgical resections. The Human Tissue Procurement Facility of the Comprehensive Cancer Center of Case Western Reserve University and University Hospitals of Cleveland provided ACF samples (P30 CA43703). To establish colorectal cancer xenograft samples, we implanted fresh colorectal cancer specimens from surgical resections to scid mice dorsally. We collected the xenografts when they grew to $>1 \mathrm{~cm}$ in diameter. We extracted genomic DNA with a phenol-chloroform procedure. We extracted total RNA by using the TRIZOL Reagent (Invitrogen).

Methylation analysis. We carried out bisulfite modification of genomic DNA and PCR amplifications as described previously ${ }^{5}$. For bisulfite sequencing analysis, we amplified bisulfite-modified genomic DNA by using primers not specific for methylation status. We cloned the PCR products into pCR2.1 TOPO and sequenced. Primer sequences and conditions for MSP and bisulfite sequencing PCR are available on request.

RT-PCR analysis. We reverse-transcribed total RNA and carried out PCR as previously described ${ }^{5}$. We carried out real-time RT-PCR as described ${ }^{21}$. Primer sequences and conditions are available on request.

Cloning. We isolated full-length cDNAs for SFRP genes, WNT1, LEF1, wildtype CTNNB1 and mutant CTNNB1 ( $\beta$-catenin $\triangle 45$ ) by using RT-PCR and cloned them into pCR2.1 TOPO vector (Invitrogen). After sequences were verified, we cut out the fragments of SFRP genes, WNT1 and LEF1 with EcoRI and ligated with EcoRI-digested pCMV-HA (Clontech) or pcDNA3.1/HisA (Invitrogen). We cut the CTNNB1 fragments with $B a m \mathrm{HI}$ and $\mathrm{XbaI}$ and ligated them with BamHI and XbaI-digested pcDNA3.1/HisC (Invitrogen). Primer sequences and conditions are available on request.

Reporter assay. We plated $5 \times 10^{4}$ cells per well on 24 -well tissue culture plates $24 \mathrm{~h}$ before transfection. We transfected cells in each well with $100 \mathrm{ng}$ of pGL3OT, a TCF-LEF-responsive reporter, or pGL3-OF, a negative control with a mutated TCF-LEF binding site; pCMV-HA-SFRPs with or without an empty vector to a total amount of $100 \mathrm{ng}$; and $2 \mathrm{ng}$ of pRL-CMV vector using FuGENE6 (Roche). For cotransfection of pCMV-HA-SFRPs and pcDNA3.1/HisA-WNT1 or $\beta$-catenin or LEF1, we transfected cells with $50 \mathrm{ng}$ of each vector plus reporter assay genes. After $48 \mathrm{~h}$, we measured luciferase activities in a luminometer (BD Biosciences) and normalized the data for the background Renilla luciferase activities by using the Dual Luciferase Reporter Assay system (Promega) according to the manufacturer's instructions.

Transfection, western-blot analysis and immunofluorescence. For transfection, we plated $3 \times 10^{5}$ cells per well on 6-well tissue culture plates $24 \mathrm{~h}$ before experiments and transfected them with $4 \mu \mathrm{g}$ total of a control plasmid and/or expression vectors using Lipofectamine 2000 (Invitrogen). For western-blot analysis, we collected cells after $48 \mathrm{~h}$, separated freeze-thaw lysates of cells by SDS-PAGE and blotted them onto nitrocellulose membranes. We used antibodies against hemagglutinin (Santa Cruz), Xpress Epitope (Invitrogen) and $\beta$-catenin (Transduction Laboratories) at 1:200, 1:5,000 and 1:500 dilutions for immunoblot analysis. Immunofluorescence studies were carried out as described ${ }^{30}$.

a

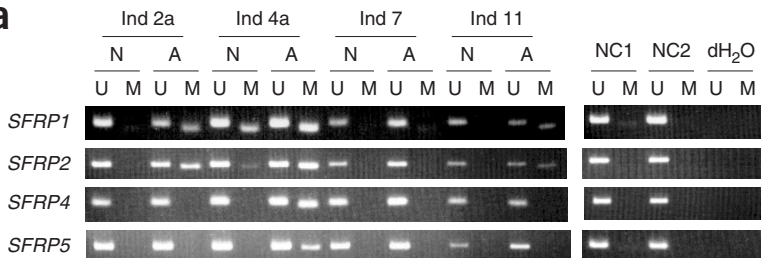

b

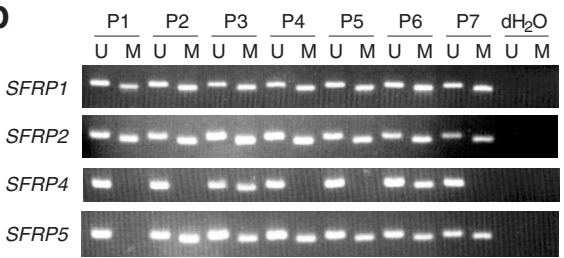

C

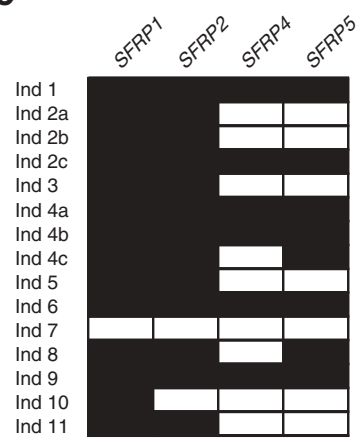

d

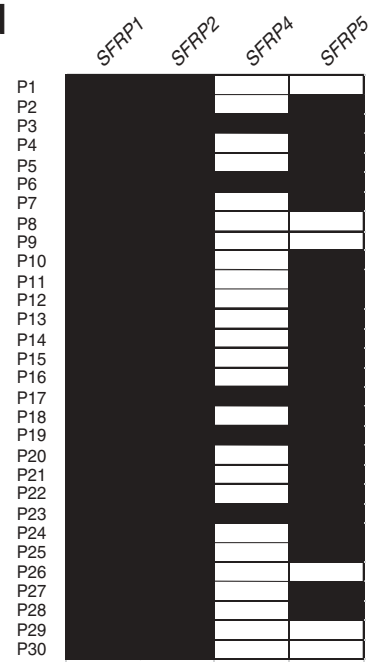

Figure 6 Methylation analysis of SFRP genes in ACF and colorectal adenoma samples. (a) Examples of MSP analysis in ACF (A) and paired normal colon (N) samples. Gene symbols are indicated on the left. Numbers identifying individuals are listed at top. M, PCR products for methylation-specific primers; $\mathrm{U}$, products with unmethylated-specific primers. MSP analysis in colon samples from healthy individuals (NC) are also shown. (b) Examples of MSP analysis in adenomas. (c) Summary of methylation analysis of SFRP genes in 15 ACF lesions from 11 individuals with colorectal cancer. Gene symbols are indicated at the top, and each row represents an ACF from an individual lesion. Filled boxes and open boxes indicate methylation and no methylation, respectively. (d) Summary of methylation analysis of SFRP genes in 30 colon adenomas. Gene symbols are indicated at the top, and each row represents an adenoma sample. Filled boxes and open boxed indicate methylation and no methylation, respectively. 
Northern-blot analysis. We resolved $10 \mu \mathrm{g}$ of total RNA by agarose gel electrophoresis in the presence of formaldehyde and hybridized it with MYC or GAPD coding region probes amplified by RT-PCR as described above.

Colony formation assay. For selection and colony formation assay, we transfected cells with pcDNA3.1/HisA-SFRPs using Lipofectamine 2000 as described above, stripped and plated them on 100-mm tissue culture dish $24 \mathrm{~h}$ after transfection and selected them for $14 \mathrm{~d}$ with $0.6 \mathrm{mg} \mathrm{m}^{-1}$ geneticin (Invitrogen).

Flow cytometry. We carried out flow cytometry analysis as described ${ }^{21}$. We fixed cells transfected either with each SFRP vector or with an empty vector, treated them with $2 \mathrm{mg} \mathrm{ml}^{-1}$ of RNase for $30 \mathrm{~min}$ at $37^{\circ} \mathrm{C}$ and stained them in $50 \mu \mathrm{g} \mathrm{ml}^{-1}$ propidium iodide solution. We used a FACScan flow cytometer (Becton Dickinson) for analysis.

Note: Supplementary information is available on the Nature Genetics website.

\section{ACKNOWLEDGMENTS}

We thank B. Vogelstein for providing pGL3-OT and pGL3-OF reporters and for comments on the manuscript; S. Eguchi, B. Reese, K. Ogi and K. Akino for technical advice; and K. Bender for technical support. S.D.M. is an Investigator in the Howard Hughes Medical Institute and supported by the National Cancer Institute. T.P.P. is supported by grants from the US Public Health Service and the National Cancer Institute. This work was supported by a grant from the National Institute of Environmental Health Services.

\section{COMPETING INTEREST STATEMENT}

for details.

Received 2 December 2003; accepted 11 February 2004

Published online at http://www.nature.com/naturegenetics/

1. Fodde, R., Smits, R. \& Clevers, H. APC, signal transduction and genetic instability in colorectal cancer. Nat. Rev. Cancer 1, 55-67 (2001).

2. Korinek, V. et al. Constitutive transcriptional activation by a $\beta$-catenin-Tcf complex in $\mathrm{APC}^{-/}$colon carcinoma. Science 275, 1784-1787 (1997)

3. Morin, P.J. et al. Activation of $\beta$-catenin-Tcf signaling in colon cancer by mutations in $\beta$-catenin or APC. Science 275, 1787-1790 (1997).

4. Liu, W. et al. Mutations in AXIN2 cause colorectal cancer with defective mismatch repair by activating $\beta$-catenin/TCF signaling. Nat. Genet. 26, 146-147 (2000).

5. Suzuki, H. et al. A genomic screen for genes upregulated by demethylation and histone deacetylase inhibition in human colorectal cancer. Nat. Genet. 31, 141-149 (2002).

6. Finch, P.W. et al. Purification and molecular cloning of a secreted, Frizzled-related antagonist of WNT action. Proc. Natl. Acad. Sci. USA 94, 6770-6775 (1997).

7. Melkonyan, H.S. et al. SARPs: a family of secreted apoptosis-related proteins. Proc.
Natl. Acad. Sci. USA 94, 13636-13641 (1997).

8. Rattner, A. et al. A family of secreted proteins contains homology to the cysteine-rich ligand-binding domain of frizzled receptors. Proc. Natl. Acad. Sci. USA 94 2859-2863 (1997).

9. Xu, Q., D'Amore, P.A. \& Sokol, S.Y. Functional and biochemical interactions of Wnts with FrzA, a secreted Wnt antagonist. Development 125, 4767-4776 (1998).

10. Chang, J.T. et al. Cloning and characterization of a secreted frizzled-related protein that is expressed by the retinal pigment epithelium. Hum. Mol. Genet. 8, 575-583 (1999)

11. Smith, K. et al. Up-regulation of macrophage WNT gene expression in adenoma-carcinoma progression of human colorectal cancer. Br. J. Cancer 81, 496-502 (1999).

12. Dimitriadis, A. et al. Expression of WNT genes in human colon cancers. Cancer Lett. 166, 185-191 (2001).

13. Holcombe, R.F. et al. Expression of WNT ligands and Frizzled receptors in colonic mucosa and in colon carcinoma. Mol. Pathol. 55, 220-226 (2002).

14. Jones, S.E. \& Jomary, C. Secreted Frizzled-related proteins: searching for relationships and patterns. Bioessays 24, 811-820 (2002).

15. Lin, K. et al. The cysteine-rich frizzled domain of FRZb-1 is required and sufficient for modulation of WNT signaling. Proc. Natl. Acad. Sci. USA 94, 11196-11200 (1997).

16. Bafico, A. et al. Interaction of frizzled related protein (FRP) with WNT ligands and the frizzled receptor suggests alternative mechanisms for FRP inhibition of WNT signaling. J. Biol. Chem. 274, 16180-16187 (1999).

17. Dennis, S., Aikawa, M., Szeto, W., D'Amore, P.A. \& Papkoff, J. A secreted frizzled related protein, FRZA, selectively associates with WNT-1 protein and regulates WNT1 signaling. J. Cell Sci. 112, 3815-3820 (1999).

18. Uren, A. et al. Secreted frizzled-related protein-1 binds directly to Wingless and is a biphasic modulator of WNT signaling. J. Biol. Chem. 275, 4374-4382 (2000).

19. Rhee, I. et al. DNMT1 and DNMT3b cooperate to silence genes in human cancer cells. Nature 416, 552-556 (2002)

20. Bachman, K.E. et al. Histone modifications and silencing prior to DNA methylation of a tumor suppressor gene. Cancer Cel/ 3, 89-95 (2003).

21. Toyota, M. et al. Epigenetic inactivation of CHFR in human tumors. Proc. Natl. Acad. Sci. USA 100, 7818-7823 (2003)

22. Hovanes, K. et al. $\beta$-catenin-sensitive isoforms of lymphoid enhancer factor- 1 are selectively expressed in colon cancer. Nat. Genet. 28, 53-57 (2001).

23. Behrens, J. et al. Functional interaction of an axin homolog, conductin, with $\beta$ catenin, APC, and GSK3ß. Science 280, 596-599 (1998).

24. Cong, F., Schweizer, L., Chamorro, M. \& Varmus, H. Requirement for a nuclear function of $\beta$-catenin in WNT signaling. Mol. Cell. Biol. 23, 8462-8470 (2003).

25. van Noort, M., Meeldijk, J., van der Zee, R., Destree, O. \& Clevers, H. Wnt signaling controls the phosphorylation status of $\beta$-catenin. J. Biol. Chem. 277, 17901-17905 (2002)

26. He, T.C. et al. Identification of c-MYC as a target of the APC pathway. Science $\mathbf{2 8 1}$ 1509-1512 (1998)

27. Siu, I.-M. et al. The identification of monoclonality in human aberrant crypt foci. Cancer Res. 59, 63-66 (1999).

28. Jen, J. et al. Molecular determinants of dysplasia in colorectal lesions. Cancer Res. 54, 5523-5526 (1994)

29. Rhee, C.S. et al. WNT and frizzled receptors as potential targets for immunotherapy in head and neck squamous cell carcinomas. Oncogene 21, 6598-6605 (2002).

30. Rountree, M.R., Bachman, K.E. \& Baylin, S.B. DNMT1 binds HDAC2 and a new corepressor, DMAP1, to form a complex at replication foci. Nat. Genet. 25, 269-277 (2000) 